\title{
Reference Modulation for Calibrated Measurements of Tag Backscatter
}

\author{
Daniel G. Kuester*†, Student Member, IEEE, David R. Novotny*, Jeffrey R. Guerrieri*, \\ Randal H. Direen*, and Zoya Popović ${ }^{\dagger}$, Fellow, IEEE \\ * U.S. Department of Commerce, National Institute of Standards and Technology, Boulder, CO, USA 80304 \\ \{daniel.kuester, david.novotny, jeffrey.guerrieri, randy.direen\} at nist.gov \\ $\dagger$ University of Colorado, Department of Electrical, Computer, and Energy Engineering, Boulder, CO, USA 80309 \\ \{daniel.kuester, zoya.popovic\} at colorado.edu
}

\begin{abstract}
This paper presents an approach for calibrating backscattering measurements from 860-960 MHz Ultra-High Frequency Radio Frequency Identification (UHF RFID) tags. An Sparameter model is formulated to relate diode switch and antenna input circuit parameters with the scattering performance of the calibration device. Measurements of modulated backscattered power agree with the model to within $\pm 0.1 \mathrm{~dB}$. Tag backscatter measurements can then be calibrated by comparing them to the reference signal. In an example testbed, the expanded uncertainty of these measurements is estimated to be $\pm 0.4 \mathrm{~dB}$, compared with uncertainties worse than $-0.9 \mathrm{~dB},+1.2 \mathrm{~dB}$ for methods that calibrate against radar cross section (RCS) standards in the same testbed.
\end{abstract}

\section{INTRODUCTION}

Continued technical advances in RFID systems are allowing passive transponders ("tags") to operate with increasingly lower powers received from transceivers ("readers") in UHF $860-960 \mathrm{MHz}$ bands. This trend enables operation at greater range, but also means that backscattered replies from tags are becoming fainter. If this trend continues, existing interference effects [1] will be made worse, and reader detection of tags may become a dominant constraint on communication in practical deployments [2]. Unfortunately, performance test standards are immature for tags, and do not yet exist for readers. The lack of strong test standards may add effort and expense to the designing robust inventory tracking systems.

System operation, in contrast, has been more extensively standardized. Typical commercial systems comply with the EPC Global "UHF Class 1 Gen 2" and ISO 18000-6C standards, which outline the operation and parameters of the halfduplex protocol used for UHF RFID. In the forward link, a reader transmits a modulated carrier towards a tag field, which harvests the incident energy to supply power for communication and processing circuitry. Each tag responds by timevarying its antenna load impedance, encoding information in the backscattered carrier. This modulated backscattering implementation, known as load modulation, forms the return link. Similar processes have been used for antenna measurements [3] and espionage [4].

U.S. GOVERNMENT WORK - Not subject to U.S. copyright.
Existing test standards leave significant unresolved challenges, particularly in the return link. They do not yet address either reader sensitivity or interference rejection, though previous work has suggested that reader sensitivity is becoming a more significant system range constraint [2], and that readers are more vulnerable to interference than tags [1]. Standard ISO 18047-6 [5] prescribes a tag backscattering performance test characterized as the difference between the radar cross section values between its two load modulation states. The prescribed test method calibrates measurements of tag backscattering against the change in received power from adding a thin rod to the test environment.

The measurement uncertainty of one test performed this way was estimated to be approximately $2 \mathrm{~dB}$ [6]. The current ISO/IEC 18047-6 method introduces further systemic error by directly subtracting power quantities, neglecting phase effects, though existing works show how phase can be considered correctly [7][8][9]. Adding and removing the entire thin rod calibration standard introduces systemic error via structuralmode modulation, which interacts with multipath in the test environment differently [2] than tags' antenna-mode modulation [7][8]. The use of such an electrically small calibration target requires faith in the accuracy of the analysis used to compute its RCS, which makes the measurement result not traceable to fundamental physical standards of any national metrology laboratory. These errors may make measurement results challenging to repeat between different testbeds, and as a result some parties may choose not to undertake the expense of running the tests.

To address these shortcomings, a new model and calibration device for traceable, more accurate measurements of backscattered tag power are proposed in this paper. The contributions are organized as follows:

- Section II introduces the model, based on S-parameters;

- Section III shows how a calibration device can be realized with a horn antenna and a laboratory switch, and characterized with the developed model parameters;

- Section IV presents the technique for calibrating tag backscatter with the calibration device;

- Section V shows a calibrated tag backscatter measurement with the new model and device. 

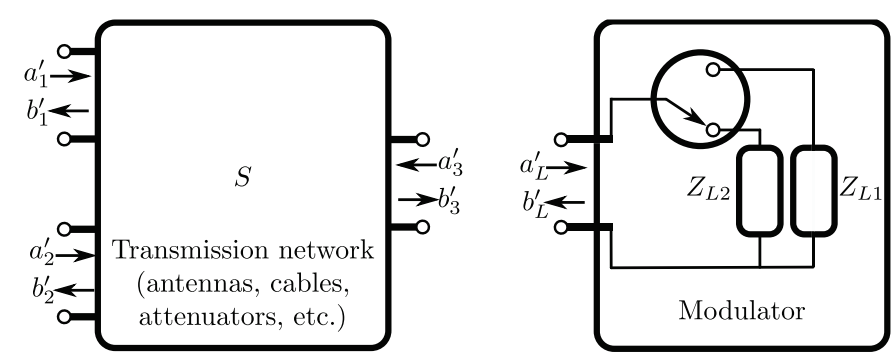

(a)

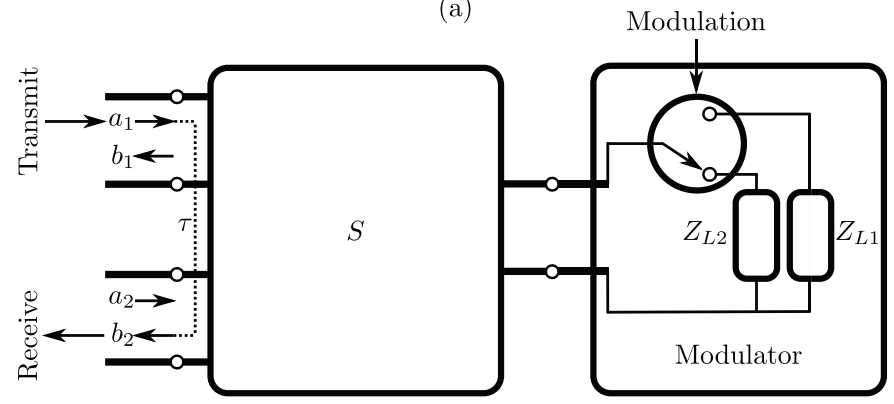

(b)

Fig. 1. Reference backscattering model illustrating S-parameters with the modulator (a) disconnected and (b) connected to the transmission network. When connected, a transmitter delivers a traveling wave $a_{1}$ into port 1 of an arbitrary transmission system $S$, which is described in (a). The reflection coefficient presented to waves arriving at port 3 switches between $\Gamma_{L} \rightarrow \Gamma_{L 1}$ (the $Z_{L 1}$ state) and $\Gamma_{L} \rightarrow \Gamma_{L 2}$ (the $Z_{L 2}$ state). Reflection back to port 2 is the traveling wave $b_{2}$. The transmit-to-receive transmission coefficient in a tag state is $\tau=b_{2} / a_{1}$.

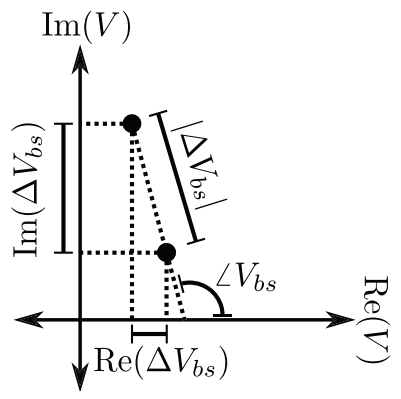

Fig. 2. Illustration of demodulated $\Delta V_{b s}$ on the complex (IQ) plane received from a reference backscatterer. The signal is shown in rectangular coordinates as $\operatorname{Re}\left(\Delta V_{b s}\right)+j \operatorname{Im}\left(\Delta V_{b s}\right)$, and in polar form as magnitude and phase.

\section{LINK AND LOAD MODULATION MODEL}

\section{A. Derivation}

The proposed model for transmission and backscattering between an interrogator, and modulator (realized in subsequent sections as a calibration device) is illustrated in Fig. 1. The linear and reciprocal transmission network is shown as $S$, where interrogation signals are incident at port 1 , backscattering is measured at port 2, and the backscatter modulator loads port 3. This network can incorporate any cables, antennas, linear propagation effects, or other linear circuit elements in the testbed.

If the two networks are disconnected (Fig. 1a), transmission coefficients between the loaded ports in the reciprocal trans- mission are $S_{23}=S_{32}=b_{3}^{\prime} / a_{2}^{\prime}$ and $S_{31}=S_{13}=b_{3}^{\prime} / a_{1}^{\prime}$, and can be measured conveniently with a network analyzer if they are connectorized. Port 3 bisects the reference backscatter standard. The reflection coefficients of the backscattering antenna and the modulator are $S_{33}=b_{3}^{\prime} / a_{3}^{\prime}$ and $\Gamma_{L}=b_{L}^{\prime} / a_{L}^{\prime}$, respectively. These coefficients are measurable with a network analyzer by disconnecting the reference backscattering antenna from the modulator.

When the two networks are connected (as in Fig. 1b), an incident carrier wave from port 1 arrives at the load modulator, which is time-varying its impedance between two states. Reflections off of this interface arrive at port 2 with two corresponding states that have in-phase and quadrature $(I Q)$ voltage components illustrated in Fig. 2.

The modulated signal $\Delta V_{b s}$ is related to a transmit signal $V_{t x}$ in an arbitrary environment as follows. The traveling waves $a_{1}$ and $b_{2}$ in Fig. $1 \mathrm{~b}$ are $a_{1}=V_{t x} / Z_{0}^{1 / 2}$ and $b_{2}=$ $V_{b s} / Z_{0}^{1 / 2}$, with purely real port impedances $Z_{0}$. An expression for the received wave $b_{2}$ in this system with port 3 loaded by the tag is [10]

$$
b_{2}=\left(S_{21}+\frac{S_{31} S_{23} \Gamma_{L}}{1-S_{33} \Gamma_{L}}\right) a_{1}+\left(S_{22}+\frac{S_{32} S_{23} \Gamma_{L}}{1-S_{33} \Gamma_{L}}\right) a_{2} .
$$

Assuming that Port 2 is not transmitting $\left(a_{2}=0\right)$, the transmission coefficient $\tau=b_{2} / a_{1}$ between the transmit and receive ports becomes

$$
\tau=S_{21}+\frac{S_{31} S_{23} \Gamma_{L}}{1-S_{33} \Gamma_{L}}
$$

This relationship has been observed for other two-port modulated scattering models [11] [12]. If the modulator load alternates between $\left\{Z_{L 1}, Z_{L 2}\right\}$, corresponding to reflection coefficients $\Gamma_{L} \rightarrow\left\{\Gamma_{L 1}, \Gamma_{L 2}\right\}$, then the change in the transmission coefficient $\Delta \tau$ will be

$$
\Delta \tau=\frac{\Delta V_{b s}}{V_{t x}}=S_{31} S_{23} \frac{\Gamma_{L 2}-\Gamma_{L 1}}{\left(1-S_{33} \Gamma_{L 2}\right)\left(1-S_{33} \Gamma_{L 1}\right)} .
$$

The term on the right gives a convenient definition for a return modulation depth

$$
M=\frac{\Gamma_{L 2}-\Gamma_{L 1}}{\left(1-S_{33} \Gamma_{L 2}\right)\left(1-S_{33} \Gamma_{L 1}\right)}=\frac{\Delta \tau}{S_{31} S_{23}},
$$

relating system behavior through the transmission network $\left(\Delta \tau, S_{31}\right.$, and $\left.S_{23}\right)$ with measurable parameters inside the backscatter reference $\left(\Gamma_{L 1}, \Gamma_{L 2}\right.$, and $\left.S_{33}\right)$.

The relationship in (3) expressed with received modulation power $P_{b s}$ between the two switched states at the reader, transmission link losses $\left|S_{31}\right|^{2}$ and $\left|S_{23}\right|^{2}$, and the carrier transmit power $P_{t x}$ is ${ }^{\dagger}$

$$
\frac{P_{b s}}{P_{t x}}=\frac{\left|\Delta V_{b s}\right|^{2} / 2 Z_{0}}{\left|V_{t x}\right|^{2} / 2 Z_{0}}=|\Delta \tau|^{2}=\left|S_{31}\right|^{2}\left|S_{23}\right|^{2}|M|^{2} .
$$

$\dagger$ Power quantities given in this model are available power. Finding power delivered into a receiver or from a source must additionally account for mismatch between the impedances on either side of the network interface. 
If the input propagation environment (an antenna or transmission line) is matched to an instrument's characteristic impedance $Z_{0}\left(S_{33}=0\right)$, then the modulation index in (5) expressed in terms of power $|M|^{2}$ is bounded by $|M|^{2}<4$. This is the case of two modulator states with reflection coefficients of with magnitude 1 that are out of phase. If $S_{33} \neq 0,|M|$ can become quite large or small, but energy is conserved because the mismatch reduces the magnitude of the transmission coefficients $S_{31}$ and $S_{23}$.

This relationship applies most directly to bistatic measurement antenna configurations, with separate transmit and receive antennas corresponding to ports 1 and 2 of Fig. 1. It can also apply to monostatic configurations if $S$ incorporates a circulator.

\section{B. Comparison with other scattering models}

The best-known modulated tag scattering characteristic is known as either a "differential" (e.g. [7] [13]) or "modulated" ([14]) radar cross section (RCS). These terms are also sometimes used interchangeably with the terms "delta RCS" or " $\triangle$ RCS" used in ISO 18047-6, though this term may cause confusion over the role of phase in tag scattering.

The modulated RCS model is formulated with power waves [7][13][14][15], which should not be confused with the Sparameter traveling waves used in this paper. The power wave parameters are convenient for chip-to-antenna matching in tag design. In metrology, however, power waves are not suitable for extracting physical parameters like impedance from measured data [16], making them unsuitable for calibration purposes.

A more recent model of tag scattering based on reciprocity and impedance (Z-) parameters was introduced in [8], and can be shown to be equivalent to the work presented here. Like ours, this model requires knowledge of a chip and antenna circuit parameters. The choice of S-parameters instead Zparameters was motivated by practical because far-field propagation effects between antennas are understood in terms of traveling waves (rather than mutual impedances), and because microwave instrumentation uncertainty is expressed in terms of S-parameters.

This paper is not intended to offer a "black box" characterization like RCS for tag testing. Direct application of the model presented here to tag characterization would require chip and antenna parameters either by prior knowledge or destructive disassembly of a tag.

\section{An idealized free-space example}

Though the goal of this work is not to present RCS measurements, the relationship between the S-parameter scattering model and modulated RCS can give some insight. As a simple test case, consider an idealized monostatic system in an anechoic environment. The backscattering and detection antennas are well-matched and co-polarized, and we assume there are no near-field effects. Measurement and backscattering antennas have gains $G$ and $G_{b s}$ towards each other. With antenna reciprocity $\left(\left|S_{31}\right|^{2}=\left|S_{23}\right|^{2}\right.$ in (5)) and the Friis transmission equation, the backscattered power is

$$
\frac{P_{b s}}{P_{t x}}=G^{2} G_{b s}^{2}\left(\frac{\lambda}{4 \pi R}\right)^{4}|M|^{2},
$$

where $R$ is the range between the measurement and backscattering antennas and $\lambda$ is the free-space carrier wavelength. This makes the same assumptions as the radar equation, and has the same form, so it can be related to the modulated radar cross section $\sigma_{m o d}$ (see for example [14]) used for tags:

$$
\sigma_{\text {mod }}=G_{b s}^{2}|M|^{2} \frac{\lambda^{2}}{4 \pi} .
$$

Relating the S-parameter network model to modulated RCS in free space therefore requires additional information about the gain of the backscattering antenna.

\section{A REFERENCE SOURCE OF BACKSCATTER MODULATION}

The model in the previous section suggests that a carefully characterized source of reference modulation with a known modulation depth $(|M|)$ and measurements of link losses ( $\left|S_{31}\right|$ and $\left.\left|S_{23}\right|\right)$ are sufficient to predict the backscattered reference power. This section introduces a device that can be characterized to serve this purpose, and how it can be validated as an accurate reference for calibrating tag backscatter.

\section{A. Calibrated signal generation}

The modulator shown in Fig. 3 realizes the model in Fig. 1. The switch has a nominal $20 \mathrm{~ns}$ rise/fall time to within $10 \%$ of steady state, which is fast enough to emulate the maximum $640 \mathrm{kHz}$ symbol rate by tags compliant with ISO/IEC 18000$6 \mathrm{C}$.

A standard gain horn with a measured gain of $6.69 \mathrm{dBi}$ at $1 \mathrm{GHz}$ is the reference backscattering antenna. It has return loss greater than $10 \mathrm{~dB}$ across $860-960 \mathrm{MHz}$, corresponding to $\left|S_{33}\right|$.

Instruments with $50 \Omega$ input impedance serve both as a matched load modulation state (with $\left|\Gamma_{L 2}\right| \approx 0$ ), and to allow measurements of interrogation signal link losses. A network analyzer makes a convenient matched load for characterizing the device, and a power meter is subsequently used to measure received interrogation power. An additional $3 \mathrm{~dB}$ pad between the switch output and the matched load attenuates reflections between the horn and the instrument. The other switch load is a short, for $\left|\Gamma_{L 1}\right| \approx 1$, though the actual $\left|\Gamma_{L 1}\right|$ is approximately $2 \mathrm{~dB}$ smaller because of switch insertion loss.

With $\left|\Gamma_{L 2}-\Gamma_{L 1}\right| \approx 1$, and the backscatter antenna approximately matched for small $\left|S_{33}\right|$, the anticipated $|M|$ is near $0 \mathrm{~dB}$.

\section{B. Measuring modulation depth}

To become suitable for calibrations with reference backscatter, the modulation depth must be measured accurately. Equation (4) gives a choice between two sets of parameters that can be measured with a network analyzer: "wireless" 


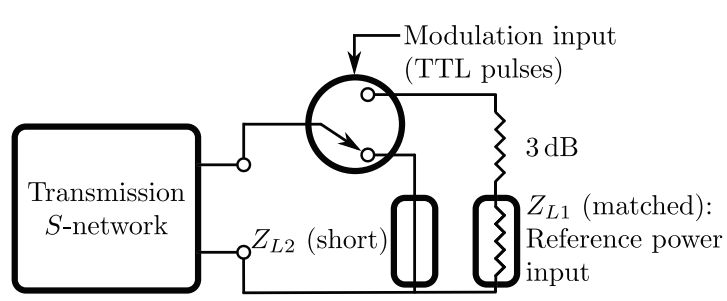

(a)

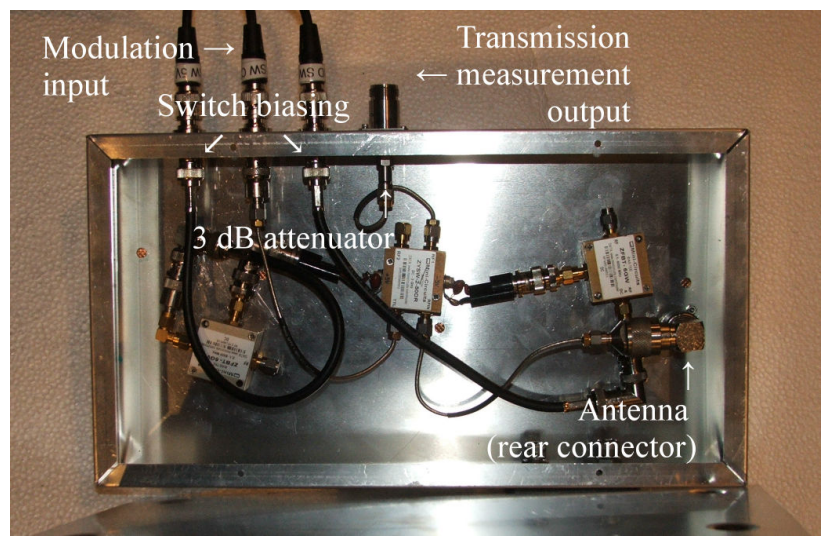

(b)

Fig. 3. Simplified schematic (a) and realization (b) of a modulation switch for reference backscatter calibrations. The load $Z_{L 1}$ is intended to connect with a matched $50 \Omega$ instrument such as a power sensor or network analyzer, to measure power delivered to the backscatter reference and serve as a matched reflection state for modulation. The device is mounted in a $33 \mathrm{~cm} \times 18 \mathrm{~cm}$ $\times 5 \mathrm{~cm}$ shielded box with $\pm 5 \mathrm{~V}$ DC biasing inputs, and bias tees to improve $\mathrm{DC}$ to RF isolation.

measurements of $S_{23}, S_{31}$, and $\Delta \tau$, or reflection coefficient measurements of $\Gamma_{L 2}, \Gamma_{L 1}$, and $S_{33}$. In either case, the modulation state of the modulator is fixed with a DC voltage supply during measurement.

One advantage of calibrating $M$ from measurements of $\Gamma_{L 2}, \Gamma_{L 1}$, and $S_{33}$ directly is that (to first order) measurement dynamic range is not reduced by moving the reference backscatter antenna in an anechoic test environment. Further, $\Gamma_{L 2}$ and $\Gamma_{L 1}$ can be measured with phase-stable cables near the network analyzer more accurately than with the long cables that are necessary to measure reflection coefficients of objects inside the test chamber.

Calibrating $M$ from $\Delta \tau$ and measurements of propagation losses $S_{23}$ and $S_{31}$ has different advantages. Transmission measurements of propagation losses can have smaller uncertainties than the reflection measurements in the first approach, but any motion in the long cables may introduce additional phase errors. This calibration also needs fewer measurements, which may reduce the contribution of operator mistakes to measurement error.

Detailed quantitative comparison of uncertainties in these approaches will be left for future work. The following subsection will validate that either set of measurements can produce a valid characterization of $|M|$.

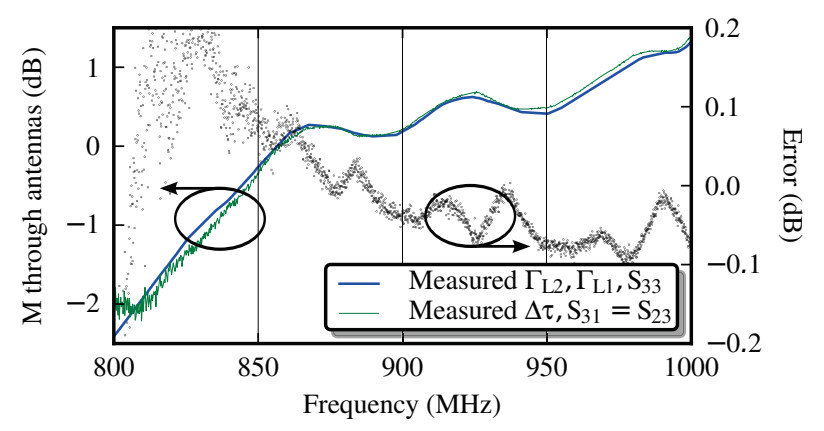

Fig. 4. Validation of the reference backscatter with a network analyzer in a semi-anechoic test environment, computed with measurements of the terms in (4). The curves agree to $\pm 0.1 \mathrm{~dB}$ over the $860-960 \mathrm{MHz}$ tag response bandwidth.

\section{Validation}

Because the model in equation (4) gives two expressions for $M$ in terms measurable network parameters, the modulation depth characterization can be validated by measuring the parameters for each with a network analyzer and comparing the results.

The validation tests, for simplicity, were performed is monostatic. The transmit and receive antenna is a commercial RFID patch with at least $10 \mathrm{~dB}$ return loss across $895-940 \mathrm{MHz}$. The change in reflection coefficient $\Delta \rho$ into the antenna was taken to be equivalent to $\Delta \tau$, with the simplification $S_{23}=S_{13}$. The anechoic environment reduces interference from outside signals, but the calibration for backscattered signal levels applies in other, more reflective environments too, if interference is below a tolerable level.

Results are shown in Fig. 4. Across the $860-960 \mathrm{MHz}$ tag response bandwidth, the two measurements of $M$ agree within $0.1 \mathrm{~dB}$. Below $860 \mathrm{MHz}$, detection antenna mismatch introduces additional noise in transmission measurements $S_{31}$ and $\Delta \rho$, because received signals are weaker.

\section{CALibration with Reference MOdulation}

\section{A. Calibration procedure}

1) Reference $|M|$ calibration: The validated characterization of the reference backscatter modulation depth $M$ from the previous section is used. In this work, tag calibrations use the modulation depth based on measurements of $\Gamma_{L 2}, \Gamma_{L 1}$, and $S_{33}$.

2) Transmission loss measurements: During tag measurements, it is impractical to measure transmission coefficients $\left|S_{31}\right|^{2}=\left|S_{13}\right|^{2}$ and $\left|S_{23}\right|^{2}=\left|S_{32}\right|^{2}$ with a network analyzer. Instead, we use power sensors to measure (1) transmitted interrogation power $P_{t x}$ available to port 1 or 2 with a coupler, and (2) power received at the output of the modulator switch, $P_{r x}^{(r e f)}$. In this paper, transmit, received (by transmission), and backscattered power from an interrogation into port $n$ are represented as $P_{t x, n}, P_{r x, n}$, and $P_{b s, n}$. Assuming the network analyzer and power sensors are similarly well-matched, power 


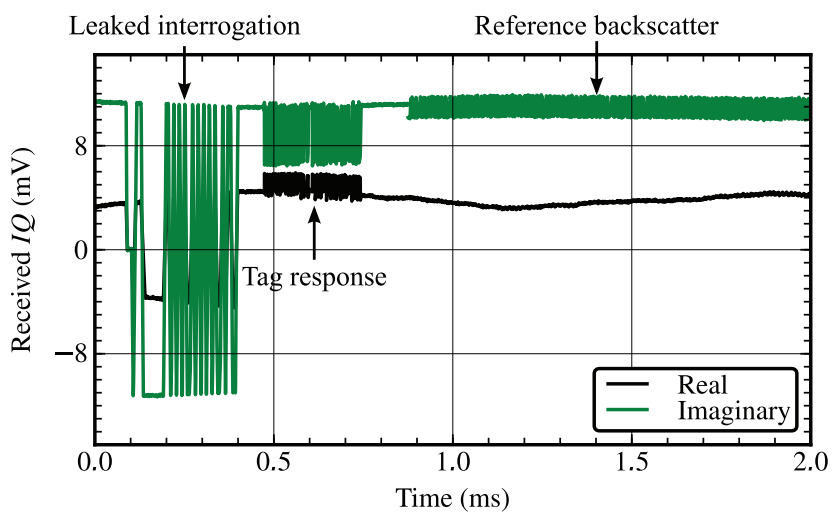

Fig. 5. A demodulated trace from a transaction at $910 \mathrm{MHz}$ with an ISO/IEC 18000-6C tag received by a spectrum analyzer. It shows leaked interrogation modulation from the forward link, the tag response from the reverse link, and reference backscatter from the calibration device introduced in this paper. In use, the reference backscatter is only turned on when it is being measured, to avoid interfering with the tag.

measurements and transmission losses are related with

$$
\left|S_{31}\right|^{2}=\left|S_{13}\right|^{2}=\frac{P_{r x, 1}^{(r e f)}}{P_{t x, 1}}
$$

and

$$
\left|S_{32}\right|^{2}=\left|S_{23}\right|^{2}=\frac{P_{r x, 2}^{(r e f)}}{P_{t x, 2}} .
$$

$P_{r x} / P_{t x, 1}$ and $P_{r x} / P_{r x, 2}$ are taken as $\left|S_{32}\right|^{2}$ and $\left|S_{31}\right|^{2}$. Both sensors are configured to measure average power during the period after the tag reply while the interrogation power is left on. This period was set to $1 \mathrm{~ms}$, which is longer than that of typically deployed readers to reduce noise by averaging.

Loss in the switch reduces the measured power compared to the available $P_{r x}^{(r e f)}$ out of the backscattering antenna. To "back out" $P_{r x}^{(r e f)}$, the full two-port scattering parameters of the switch are used to de-embed the power available out of port 3 with transfer (T-) parameters.

3) Backscattered signal measurements and processing: Backscattered power $P_{b s}^{(\text {tag,meas })}$ and $P_{b s}^{(\text {ref,meas })}$ are measured with $I Q$ demodulation on a spectrum analyzer. Example signal traces are shown in Fig. 5. The interrogation signal is an ISO/IEC 18000-6C query command, with tag and reference responses modulated at $160 \mathrm{kHz}$.

Tag responses to query requests are measured in a $240 \mu \mathrm{s}$ period, and reference backscatter in $1 \mathrm{~ms}$. Connecting the $10 \mathrm{MHz}$ frequency reference from the spectrum analyzer to the RFID interrogation source, carrier phase drifted by less than $1.3^{\circ}$ per symbol at $160 \mathrm{kHz}$ modulation, introducing negligible error to measurements of signal levels between adjacent symbols. Across entire tag or reference signal traces, however, the carrier drifts by up to $13^{\circ}$. This made separating the signal into the two states in software ("clustering") more challenging: slow drift in each of the $I$ and $Q$ components was often larger than the backscattered signal, making the straightforward histogram analysis suggested by IEEE 181 [17] unsuitable.
Instead, states are clustered by applying a Gaussian filter to the $I$ and $Q$ scattering components, and finding sharp peaks after differentiating the result. These peaks are reported when they are a local amplitude maximum; this threshold was set somewhat arbitrarily to the standard deviation of the filtered signal. The Gaussian filter is convenient here because it removes noise without shifting pulse edges; it is a simpler onedimensional implementation of Canny edge detection [18].

If $\Delta V_{I}$ and $\Delta V_{Q}$ are the differences between the averaged two backscattering state measurements of each component, $\Delta V_{b s}$ is computed as (3),

$$
\Delta V_{b s}=\left(\Delta V_{I}+j \Delta V_{Q}\right) \exp j \phi
$$

where the exponential term accounts for the arbitrariness $I$ $Q$ plane rotation. For vector voltage quantity $\Delta V_{b s}$, averaging across neighboring state transitions in different frames reduces measurement noise, potentially to below $-100 \mathrm{dBm}$.

The power in these two states, corresponding to $P_{b s}$ from (5), was calculated as

$$
P_{b s}=\frac{\left|\Delta V_{b s}\right|^{2}}{2 Z_{0}}=\frac{\left|\Delta V_{I}+j \Delta V_{Q}\right|^{2}}{2 Z_{0}},
$$

in agreement with previous work [7][9][13][19].

4) Correcting the tag backscatter measurement: Assuming bandwidth of all backscattered signals are narrow about the interrogating carrier, and that cable and antenna mismatch and losses are linear with power, the fractional power lost will be the same for both a tag and reference backscatter:

$$
\frac{P_{b s}^{(\text {ref,meas })}}{P_{b s}^{(r e f)}}=\frac{P_{b s}^{(\text {tag }, \text { meas })}}{P_{b s}^{(\text {tag })}}
$$

This can be rearranged to find "true" backscattered power received from the tag,

$$
P_{b s}^{(\text {tag })}=\frac{P_{b s}^{(\text {tag,meas })}}{P_{b s}^{(\text {ref,meas })}} P_{b s}^{(\text {ref })}
$$

Equations (5), (8), and (9) can be substituted for $P_{b s}^{(r e f)}$, so for interrogation through port 1 ,

$$
P_{b s, 1}^{(\text {tag })}=\frac{P_{b s, 1}^{(\text {tag }, \text { meas })}}{P_{b s, 1}^{(\text {ref }, \text { meas })}} \frac{P_{r x, 2}^{(r e f)}}{P_{t x, 2}} P_{r x, 1}^{(r e f)}|M|^{2} .
$$

or through port 2 ,

$$
P_{b s, 2}^{(t a g)}=\frac{P_{b s, 2}^{(\text {tag,meas })}}{P_{b s, 2}^{(\text {ref,meas })}} \frac{P_{r x, 1}^{(\text {ref })}}{P_{t x, 1}} P_{r x, 2}^{(r e f)}|M|^{2} .
$$

Notice that, like the RCS calibration in ISO 18047-6, effects of mismatch or cable losses between the receive antenna and the measurement instrument are removed in the calibration process. 


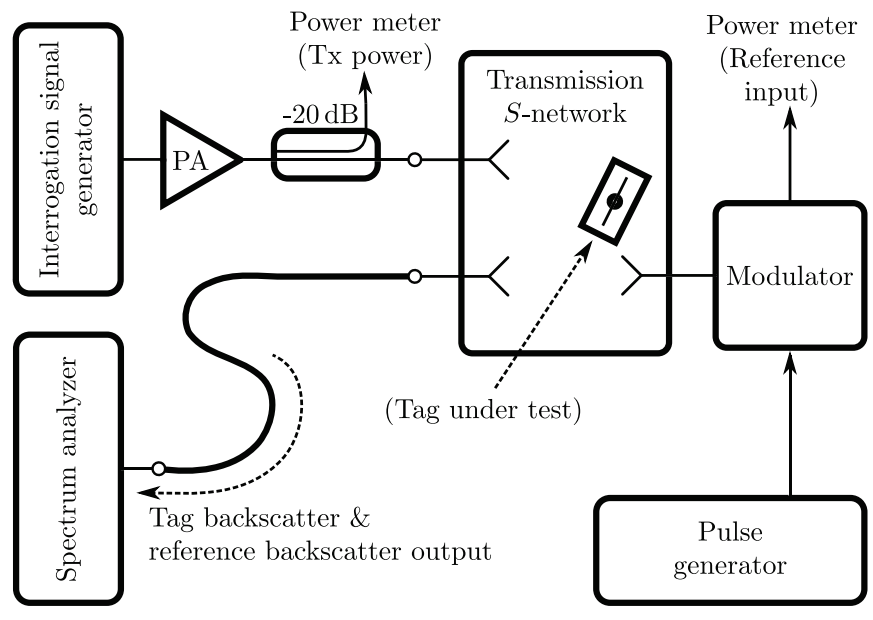

Fig. 6. Setup for calibrated tag backscattering measurements. Measurements were automated by computer via general purpose interface bus (GPIB).

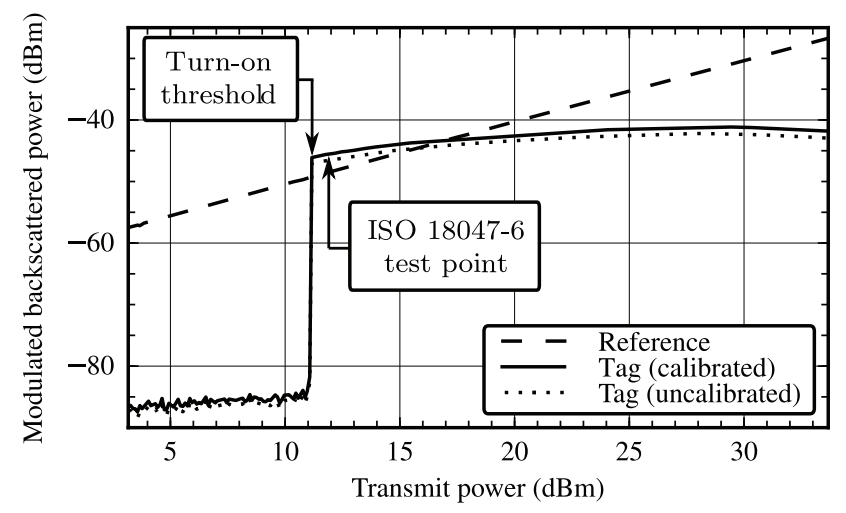

Fig. 7. Measurements of backscattered power comparing raw tag and reference signals with the calibrated measurement of the tag. The reference was switched at $160 \mathrm{kHz}$ modulation, and the tag was interrogated with signal parameters described in table I. Signals from each were measured with $I Q$ demodulation as in Fig. 8. The calibration used $M$ calculated with equation (4) from measurements of $\Gamma_{L 1}, \Gamma_{L 2}$, and $S_{33}$.

\section{EXAMPLE CALIBRATIONS}

\section{A. Calibrated tag measurements}

With the procedure established, we next present an example tag backscatter measurement, calibrated with the backscattering source. The overall test setup is summarized in Fig. 6 and Table I. Instruments are controlled with computer via general purpose interface bus (GPIB).

The parameters from Table I were used in the calibrated tag backscatter measurement shown in Fig. 7, showing highly nonlinear raw and calibrated measurements of the tag under test with the reference backscatter for comparison. Reference backscatter was turned off during measurements of the tag response, to prevent interference.

\section{B. Measurement errors and uncertainty}

To help gauge the effectiveness of the reference backscatter calibration, an uncertainty estimate is presented in Table II,
TABLE I

ISO/IEC 18000-6C TAG QUERY PARAMETERS

\begin{aligned} & \hline Reader-to-tag modulation PR-ASK \\ & Tag-to-reader modulation FM0 \\ & Tag-to-reader link rate (BLF) $160 \mathrm{kHz} \\ &$ Reader-to-tag link rate $160 \mathrm{kHz}$ (data 0) \\ & $91 \mathrm{kHz}$ (data 1) \\ & Anticollision slots (Q) 0 (no slots) \\ & Delay after tag response ${ }^{\dagger}(\mathrm{T} 2) 1 \mathrm{~ms} \\ &$ Tari $6.25 \mu \mathrm{s} \\ &$\hline Reference backscattering was measured during this period \end{aligned}

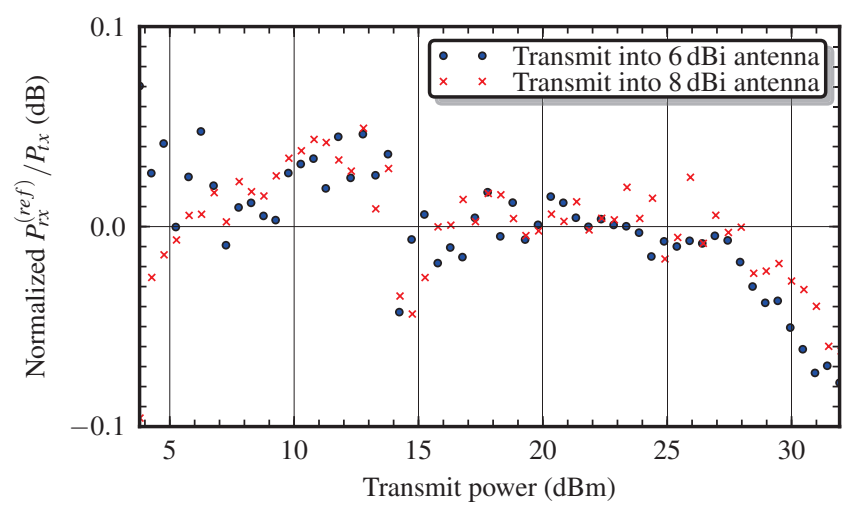

Fig. 8. Testbed linearity tests, performed by sweeping the power of the interrogating wave and measuring the reference backscattered power. The backscatter reference load-modulated $910 \mathrm{MHz}$ carrier reflections at $160 \mathrm{kHz}$ with the circuit described in Fig. 3. Deviation from linearity below $32 \mathrm{dBm}$ input power was less than $0.1 \mathrm{~dB}$.

with a comparison against other measurement approaches taken in the same testbed. Table IIb, for example, estimates uncertainty in hypothetical backscattered power measurement based on the spectrum analyzer's built-in alignment, with cable losses measured and de-embedded. The measurement in Table IIc is meant to reflect the uncertainty of a backscattering measurement that has been careful calibrated with a thin $\lambda / 2$ rod as a scattering standard, based in part on results from [2]. All of these measurements are assumed to use $I Q$ demodulation on the same spectrum analyzer.

Testbed linearity is estimated from measurements of the reference backscatter as a function of power, shown in Fig. 8. This measurement was performed with a bistatic receiver setup. Results between the two traces agreed within $\pm 0.1 \mathrm{~dB}$, which is then the estimate for linearity and noise uncertainty.

Reported values follow the methods for evaluating uncertainty expressed in [20] as closely as possible. Each error source is assumed to have a zero mean normal distribution, except multiple reflection errors that are assumed to have a U-shaped distribution. Supplied error estimates are type B, except the noise and linearity term, estimated from 8 , and the $I Q$ level measurement errors, estimated by simulation of noisy bandlimited pulses.

These uncertainty terms, because they are shown in decibels, are expressed relative to the actual measurement value. Each is listed as an expanded uncertainty, and corresponds to a fractional term $u_{i}$ that is the contribution of the individual 
TABLE II

EXPANDED UNCERTAINTY ESTIMATES (COVERAGE FACTOR 2)

(a)

Backscattered tag signal measurement

(with reference backscatter calibration)

\begin{tabular}{cc}
\multicolumn{2}{c}{ (with reference backscatter calibration) } \\
\hline$M$ calibration & $\pm 0.25 \mathrm{~dB}$ \\
Power measurements & $\pm 0.25 \mathrm{~dB}$ \\
Multiple reflections & $\pm 0.15 \mathrm{~dB}$ \\
$I Q$ level measurements & $\pm 0.05 \mathrm{~dB}$ \\
Noise and nonlinearity & $\pm 0.1 \mathrm{~dB}$ \\
\hline Expanded uncertainty & $\pm 0.4 \mathrm{~dB}$
\end{tabular}

(b)

\begin{tabular}{cc}
\multicolumn{2}{c}{$\begin{array}{c}\text { Backscattered tag signal measurement } \\
\text { (without scattering calibration) }\end{array}$} \\
\hline Transmit power measurements & $\pm 0.15 \mathrm{~dB}$ \\
Measurement cable losses & $\pm 0.1 \mathrm{~dB}$ \\
Instrument uncertainty & $\pm 0.9 \mathrm{~dB}$ \\
$I Q$ level processing & $\pm 0.15 \mathrm{~dB}$ \\
Noise and nonlinearity & $\pm 0.1 \mathrm{~dB}$ \\
\hline Expanded uncertainty & $-1.2 \mathrm{~dB},+0.9 \mathrm{~dB}$
\end{tabular}

(c)

Backscattered tag signal measurement (with ISO 18047-6 $\lambda / 2$ rod calibration)

\begin{tabular}{cc}
\multicolumn{2}{c}{ (with ISO 18047-6 $\lambda / 2$ rod calibration) } \\
\hline Reference $\lambda / 2$ rod RCS & (unknown) \\
Multiple reflections & $\pm 0.9 \mathrm{~dB}[2]$ \\
$I Q$ carrier phase drift & $\pm 0.23 \mathrm{~dB}$ \\
$I Q$ level processing & $\pm 0.15 \mathrm{~dB}$ \\
Noise and nonlinearity & $\pm 0.1 \mathrm{~dB}$ \\
\hline Expanded uncertainty & $-1.2 \mathrm{~dB},+0.9 \mathrm{~dB}$ \\
(neglecting rod RCS uncertainty) &
\end{tabular}

error source as a fraction of the measured value. The reported value in decibels is related to $u_{i}$ with

$$
u_{i}(\text { in } \mathrm{dB})=10 \log _{10}\left(1 \pm u_{i}\right) .
$$

The expanded uncertainty estimate $u_{c}$ from $n$ terms is computed with

$$
u_{c}(\text { in } \mathrm{dB})=10 \log _{10}\left(1 \pm \sqrt{\sum_{i=1}^{n} u_{i}^{2}}\right)
$$

by the law of propagation of uncertainty [20], which applies here under the assumption that errors are zero-mean and that dominant errors are normally distributed. The sensitivity coefficients are already included in the itemized uncertainties listed in Table II.

Some errors are common to all of the measurements. The noise and nonlinearity uncertainty is estimated conservatively from (8). Uncertainty in the $I Q$ measurement processing, caused by ringing or level clustering errors, is quoted as $\pm 0.05 \mathrm{~dB}$ for results based on relative backscattering signal results (like the method presented in this paper), or $\pm 0.15 \mathrm{~dB}$ for absolute measurements, based on simulation of noisy, bandlimited pulses. Spectrum analyzer measurement uncertainty is contributes to errors in all of these cases, but when a scattering standard is used, the relative measurement result from the instrument is so small that it is negligible. This contrasts with the result in Table IIb, which is a dominant $\pm 0.9 \mathrm{~dB}$ source of uncertainty estimated from the manufacturer's specification. Because the modulation of the $\lambda / 2 \operatorname{rod}$ standard in the measurement of Table IIc is performed by (very slowly) moving the standard by hand, the $\pm 13^{\circ}$ phase error becomes significant, and corresponds to a worst-case $\pm 0.23 \mathrm{~dB}$ uncertainty.

The measurement technique presented in this paper has some unique uncertainty terms. Errors introduced by calibrations of $M$ are a caused by reflection coefficient measurement uncertainty of the network analyzer and the short-open-load calibration standards. The corresponding error estimate is based on measuring verification load standards on the network analyzer after calibration, and on analysis of manufacturer uncertainty specifications. The power measurement uncertainty $\pm 0.25 \mathrm{~dB}$ is also based on analysis of the manufacturer's power sensor specifications, from analysis of correlated and uncorrelated error terms between the two power measurements of (14) and (15). The multiple reflection estimate is a conservative estimate of detuning effects on the reference backscatter antenna reflection coefficient, caused by variations in the test setup, such as variations between scattering off of different tags under test. Empirical tests showed that reorienting the antenna or moving the tag in the test zone caused errors smaller than $0.1 \mathrm{~dB}$.

\section{CONCLUSION}

The reference backscatter modulation approach demonstrated here improves signal accuracy compared to radar cross section methods, with common lab equipment available at low cost from many different vendors.

With additional effort in analysis of the uncertainty of this technique as part of RCS tag tests, the authors hope it will become useful for future versions of tag performance test standards. Further work may also prove the effectiveness of the technique in small anechoic environments to reduce test costs. Reference signals may also become practical as in sensitivity or interference rejection tests.

\section{ACKNOWLEDGEMENTS}

The authors are grateful to Prof. E. Kuester for fruitful discussions about the circuit model, and Z. Popovic acknowledges support from the Hudson Moore Jr. professorship at the University of Colorado.

The United States Department of Homeland Security Science and Technology Directorate has, in part, sponsored the production of this material with NIST, under contract HSHQDC-09-X-00305.

\section{REFERENCES}

[1] M.R. Souryal, D.R. Novotny, D.G. Kuester, J.R. Guerrieri, K.A. Remley, "Impact of RF Interference between a Passive RFID System and a Frequency Hopping Communications System in the 900 MHz ISM Band," Proc. 2010 IEEE Symp. on Electromagnetic Compatibility, pp. 495-501.

[2] D.G. Kuester, D.R. Novotny, J.R. Guerrieri, "The relative importance of the forward and reverse links in propagating UHF RFID with passive tags," Proc. 2010 IEEE Symp. on Electromagnetic Compatibility, pp. 680685.

[3] J.H. Richmond, "A Modulated Scattering Technique for Measurement of Field Distributions," IRE Trans. on Microwave Theory and Techniques, vol.3, no.4, pp. 13-15, July 1955. 
[4] A. Glinsky, Theremin: Ether Music and Espionage. Champaign, IL, USA: University of Illinois Press, 2000, pp. 259-273.

[5] Radio frequency identification device conformance test methods - Test methods for air interface communications at $860 \mathrm{MHz}$ to $960 \mathrm{MHz}$, ISO/IEC standard 18047-6, 2006.

[6] A. Pouzin, T.P. Vuong, S. Tedjini, M. Pouyet, J. Perdereau, and L. Dreux, "Determination of measurement uncertainties applied to the RCS and the differential RCS of UHF passive RFID tags," Proc. Antennas and Propagation Soc. Int. Symp., 2009, pp. 1-4.

[7] P. Nikitin and K. Rao, "Theory and measurement of backscattering from RFID tags," IEEE Antennas and Propagation Magazine, vol. 48, 2006, p. 212-218.

[8] J.C. Bolomey, S. Capdevila, L. Jofre, and J. Romeu, "Electromagnetic Modeling of RFID-Modulated Scattering Mechanism. Application to Tag Performance Evaluation," Proceedings of the IEEE, vol. 98, 2010, p. $1555-1569$.

[9] S. Skali, C. Chantepy, and S. Tedjini, "On the measurement of the delta Radar Cross Section ( $\triangle \mathrm{RCS})$ for UHF tags," 2009 IEEE International Conference on RFID, 2009, pp. 346-351.

[10] D.M. Kerns, R.W. Beatty, Basic Theory of Waveguide Junctions and Introductory Microwave Network Analysis. Oxford: Pergamon Press, 1967 , p. 108

[11] K. Seemann, F. Cilek, M. Schmidt, R. Weigel, "RFID at UHF Frequencies: The Passive Transponder Frontend Approach," Proc. Intl. Conf. on Microwaves, Radar \& Wireless Communications, 2006, pp.657-661, 22-
24 May 2006.

[12] J.C. Bolomey and F.E. Gardiol, Engineering Applications of the Modulated Scattering Technique, Boston: Artech House, 2001, pp. 35-59.

[13] F. Fuschini, C. Piersanti, F. Paolazzi, and G. Falciasecca, "Analytical Approach to the Backscattering from UHF RFID Transponder," IEEE Antennas and Wireless Propagation Letters, vol. 7, 2008, pp. 33-35.

[14] D.M. Dobkin, The RF in RFID: Passive UHF RFID in Practice, Newton, MA, USA: Newnes, 2007, pp. 19-22.

[15] K. Kurokawa, "Power Waves and the Scattering Matrix," IEEE Transactions on Microwave Theory and Techniques, vol. 13, Mar. 1965, pp. 194-202.

[16] D.F. Williams and R.B. Marks, "Comments on 'Conversions between S, $\mathrm{Z}, \mathrm{Y}, \mathrm{h}, \mathrm{ABCD}$, and $\mathrm{T}$ parameters which are valid for complex source and load impedances' [and reply]," IEEE Transactions on Microwave Theory and Techniques, vol. 43, Apr. 1995, pp. 914-915.

[17] Standard on Transitions, Pulses, and Related Waveforms, IEEE Standard 181-2003, 2003.

[18] J. Canny, "A Computational Approach to Edge Detection," IEEE Transactions on Pattern Analysis and Machine Intelligence, vol. PAMI-8, Nov. 1986, pp. 679-698.

[19] A. Pouzin, T.P. Vuong, S. Tedjini, M. Pouyet, and J. Perdereau, "Bench test for measurement of differential RCS of UHF RFID tags," Electronics Letters, vol. 46, Apr. 2010, pp. 590-592.

[20] B. Taylor, Guidelines for evaluating and expressing the uncertainty of NIST measurement results, NIST Technical Note 1297, 1994. 